\title{
Antiepileptic effects of levetiracetam in a rodent neonatal seizure model
}

\author{
Delia M. Talos ${ }^{1,5}$, Meayoung Chang ${ }^{1,6}$, Bela Kosaras ${ }^{1}$, Erin Fitzgerald ${ }^{1}$, Andrew Murphy', Rebecca Dunn Folkerth ${ }^{2,3}$ and \\ Frances E. Jensen ${ }^{1,4,7}$
}

BACKGROUND: Neonatal seizures can result in chronic epilepsy and long-term behavioral and cognitive deficits. Levetiracetam (LEV), an antiepileptic drug that binds to the synaptic vesicle protein 2A (SV2A), has been increasingly used off-label for the therapy of neonatal seizures. Preclinical data regarding the acute or long-term efficacy of LEV are lacking.

METHODS: We tested the anticonvulsant efficacy of LEV in a rat model of hypoxia-induced neonatal seizures. In addition, we evaluated the protective effects of postnatal day (P)10 LEV treatment on later-life kainic acid (KA)-induced seizure susceptibility and seizure-induced neuronal injury. Western blot and immunohistochemistry were used to assess the developmental regulation of SV2A in the rat and human brain.

RESULTS: LEV pretreatment at P10 significantly decreased the cumulative duration of behavioral and electrographic seizures at both 25 and $50 \mathrm{mg} / \mathrm{kg}$. At P40, KA-induced seizures and neuronal loss were significantly diminished in rats previously treated with LEV. LEV target SV2A is present in both neonatal rat and human brain and increases steadily to adulthood.

CONCLUSION: LEV suppressed acute seizures induced by perinatal hypoxia and diminished later-life seizure susceptibility and seizure-induced neuronal injury, providing evidence for disease modification. These results support consideration of a clinical trial of LEV in neonatal seizures.

Nistar eonatal seizures can be refractory to conventional antiepileptic drugs (AEDs) (1,2), and recently, newer-generation drugs have been considered for off-label use and/or clinical trials for this indication. Levetiracetam $((S)$ - $\alpha$-ethyl-2-oxo-1pyrrolidine acetamide; LEV) is a newer AED with more than a 10-y history of US Food and Drug Administration approval for use as adjunctive therapy for partial epilepsy and is efficacious and safe as monotherapy in adult and pediatric epilepsy syndromes $(3,4)$. LEV has a specific and unique binding target, the synaptic vesicle protein $2 \mathrm{~A}(\mathrm{SV} 2 \mathrm{~A})$, which is involved in exocytosis of synaptic vesicles by interacting with the soluble
$\mathrm{N}$-ethylmaleimide-sensitive factor attachment protein receptor complex (5). SV2A is widely expressed throughout all brain structures regardless of the neurotransmitter phenotype (6). LEV binding to SV2A impedes neurotransmitter exocytosis and can block the interaction of the SV2A protein with the actin cytoskeletal network involved in the synaptic vesicle trafficking (7). LEV has also been shown to modulate voltageoperated $\mathrm{K}^{+}$channels (8) and $\mathrm{N}$-type high-voltage-activated $\mathrm{Ca}^{2+}$ currents (9).

Previous studies in adult animal models of chronic epilepsy demonstrate that LEV is a potent anticonvulsant with longlasting antiepileptogenic effects, even as a single dose (10-13). Unlike other conventional AEDs, LEV has no negative impact on cognition and memory formation in either normal or chronically epileptic rats (14). Taken together, these properties and its unique safety profile make LEV an attractive candidate drug for seizure suppression and antiepileptogenesis in the developing brain. In addition, LEV has recently become available in an intravenous form, and several case reports and feasibility studies have documented the use of LEV in neonatal seizures (15-18). However, there is minimal preclinical evidence supporting either its acute or its long-term actions in neonatal seizure models, although of importance, animal studies have shown relative safety of LEV administration in the immature brain $(19,20)$.

In this study, we examined the efficacy of LEV pretreatment before seizure induction in our rodent model of neonatal hypoxic seizures (HSs), given that hypoxic-ischemic encephalopathy is the most common cause of neonatal seizures (1). First, we examined the efficacy of LEV as a 60 -min pretreatment on the cumulative duration of behavioral and electroencephalographic (EEG) seizures during hypoxia. Subsequent susceptibility and seizure-induced neuronal injury to the "second-hit" kainic acid (KA)-induced seizures at postnatal day (P) 40 were compared between animals receiving LEV vs. vehicle treatment at P10. The expression of SV2A protein in immature rat and human brain was also examined for target validation. 


\section{RESULTS}

LEV Suppresses Behavioral and Electrographic Seizure Activity During Neonatal Hypoxia

LEV $(10,25$, or $50 \mathrm{mg} / \mathrm{kg})$ or vehicle was administered $60 \mathrm{~min}$ before HS induction. Similar to our previously published reports, vehicle-treated pups initially responded to hypoxia with myoclonic jerks, followed by the onset of tonic-clonic head and trunk movement (21-23), with an average total duration of $341.8 \pm 28.4 \mathrm{~s}(100 \%, n=18)$. LEV treatment resulted in a significant decrease in HS duration at $25 \mathrm{mg} / \mathrm{kg}(175.0 \pm$ $33.8 \mathrm{~s}, 51.2 \%, n=8, P=0.01)$ and at $50 \mathrm{mg} / \mathrm{kg}(124.4 \pm 21.2 \mathrm{~s}$, $36.4 \%, n=19, P<0.001)$. Anticonvulsant activity was maximal at $50 \mathrm{mg} / \mathrm{kg}$, at which HSs were reduced by 63.6\% (Figure 1a).

Similarly, there was a significant decrease in the cumulative duration of ictal EEG activity during hypoxia, defined as paroxysmal, rhythmic discharges lasting more than $3 \mathrm{~s}$, as we previously published (Figure 1b,c) (24). The duration of ictal discharges was significantly less in HS groups treated with LEV at $25 \mathrm{mg} / \mathrm{kg}(175.8$ $\pm 17.6 \mathrm{~s}, 50.6 \%, n=6, P=0.041)$ and at $50 \mathrm{mg} / \mathrm{kg}(154.7 \pm 32.3 \mathrm{~s}$, $44.6 \%, n=10, P=0.005)$ as compared with vehicle-treated animals (347.1 \pm 41.7 s, $100 \%, n=9)$. Maximal anticonvulsant activity was observed at $50 \mathrm{mg} / \mathrm{kg}$, which resulted in a $55.4 \% \mathrm{HS}$ reduction.

\section{LEV Administration at P10 Attenuates Later-Life Susceptibility to KA-Induced Seizures}

We have previously shown that neonatal seizures result in increased neuronal hyperexcitability, enhanced seizure susceptibility, increased seizure-induced neuronal injury, and development of spontaneous seizures (24-26). Given the acute anticonvulsant efficacy of LEV, we next tested whether the same treatment is protective against later-life seizure susceptibility and seizureinduced neuronal death, given that this is a marker of long-lasting network excitability and correlates with later epilepsy (24). Rats that were treated with the highest LEV dose $(50 \mathrm{mg} / \mathrm{kg})$ or vehicle and exposed to either hypoxia or normoxia at P10 were subsequently treated with $\mathrm{KA}(10 \mathrm{mg} / \mathrm{kg})$ at P40. Latency to first response after KA injection (Figure 1d) was significantly shorter for the vehicle-treated HS group $(25.11 \pm 2.61 \mathrm{~min}, n=9)$ than for the control normoxic group (32.46 $\pm 1.99 \mathrm{~min}, n=13)$ or the LEV-treated HS group $(29.57 \pm 1.45 \mathrm{~min}, n=8, P=0.03)$. Similarly, seizure severity score after KA induction (Figure 1e) was higher for the vehicle-treated HS group $(3.19 \pm 0.26, n=21)$ than for the control normoxic group $(2.71 \pm 0.28, n=21)$ or the LEV-treated HS group $(2.38 \pm 0.38, n=8, P=0.03)$.
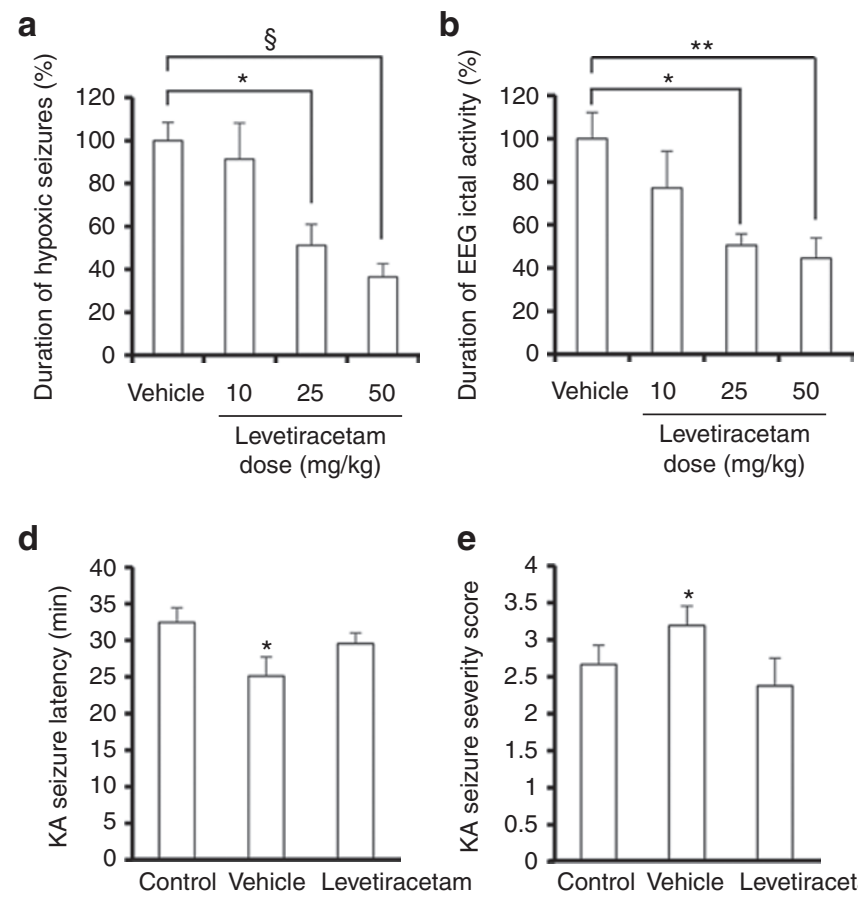

C
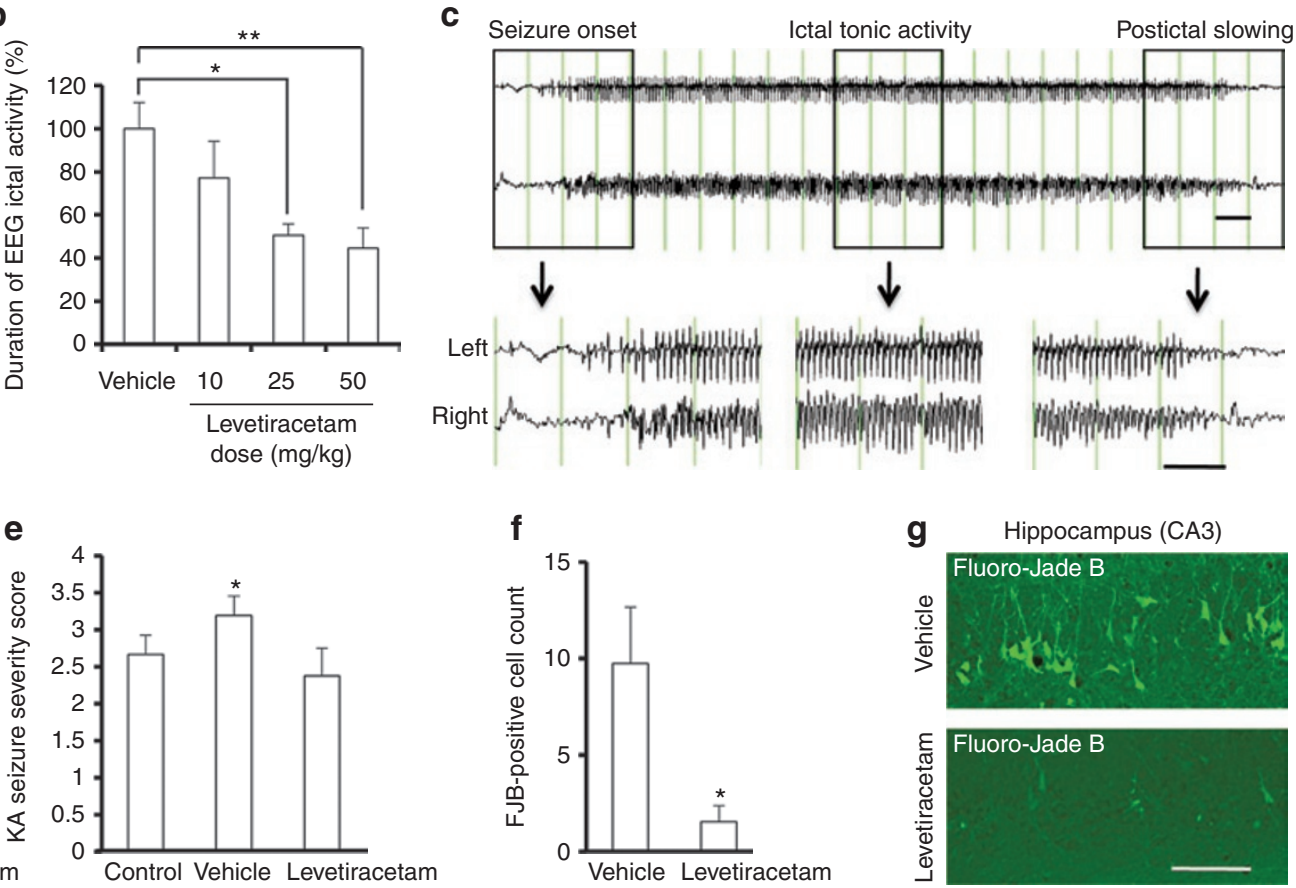

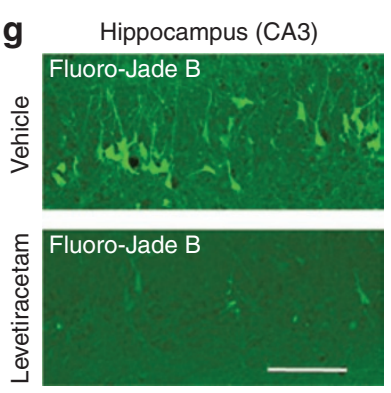

Figure 1. Levetiracetam (LEV) suppresses acute hypoxia-induced seizures in a dose-related manner and prevents later increases in seizure susceptibility and seizure-induced neuronal injury. (a) LEV treatment resulted in a significant decrease in cumulative hypoxic seizure (HS) duration at $25 \mathrm{mg} /$ $\mathrm{kg}(P=0.01)$ and at $50 \mathrm{mg} / \mathrm{kg}(P<0.001)$. Data represent the mean total duration of behavioral seizures for each LEV-treated group expressed as a percentage of the mean total duration of seizures for the paired vehicle-treated group \pm SEM. (b) The cumulative duration of ictal discharges was significantly less in HS groups treated with LEV at $25 \mathrm{mg} / \mathrm{kg}(P=0.041)$ and at $50 \mathrm{mg} / \mathrm{kg}(P=0.005)$, as compared with the vehicle-treated group. Data represent the mean total duration of seizures for each LEV-treated group expressed as a percentage of the mean total duration of seizures for the paired vehicle-treated group \pm SEM. (c) An EEG seizure was defined as paroxysmal, rhythmic, high-amplitude train of polyspikes and sharp waves lasting more than 3 s. (d) Latency to first response after kainic acid (KA) injection at P40 (pawing or wet-dog shake) was significantly shorter for the vehicle-treated HS group than for the control normoxic or LEV-treated $(50 \mathrm{mg} / \mathrm{kg}) \mathrm{HS}$ group $(P=0.03)$. (e) Seizure severity score of KA-induced seizures at P40 was higher for the vehicle-treated HS group than for the control normoxic or LEV-treated (50 mg/kg) HS group $(P=0.03)$. (f) Animals treated with LEV (50 mg/kg) $60 \mathrm{~min}$ before hypoxia at P10 showed significantly fewer Fluoro-Jade B (FJB)-positive neurons in response to KA in the hippocampal CA3 region vs. vehicletreated animals $(P=0.035)$. Of note, only animals that reached grade 3 or 4 seizures after KA injection were included in the analysis. (g) Representative FJB images of hippocampal CA3 region. Bars in c represent $1 \mathrm{~s}$. Bar in $\mathbf{g}$ denotes $100 \mu \mathrm{m}$. ${ }^{*} P<0.05,{ }^{* *} P<0.01,{ }^{5} P<0.001$. EEG, electroencephalography. 
a

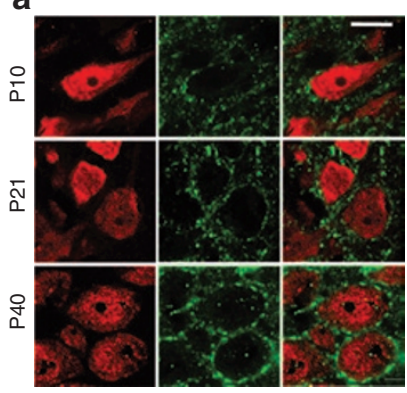

C

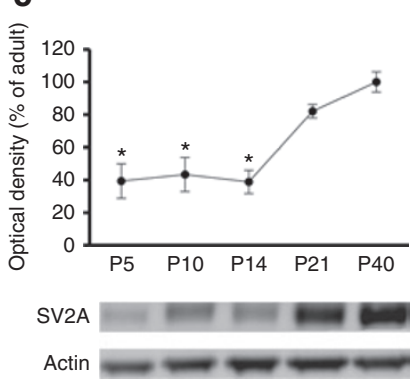

b

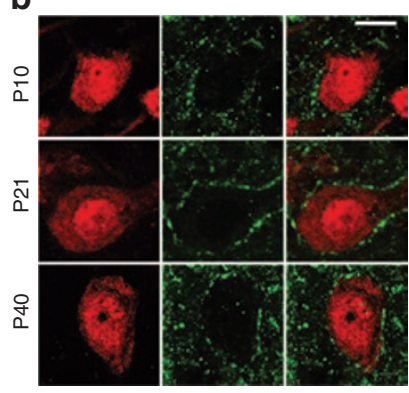

d

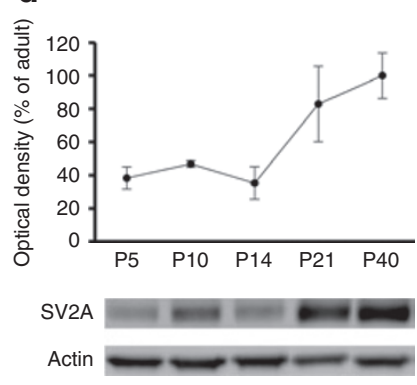

Figure 2. Presynaptic vesicle protein SV2A is developmentally regulated in rat hippocampus and neocortex. (a) Immunofluorescence double labeling with NeuN (red) and SV2A (green) demonstrated that in the rat hippocampus, SV2A was expressed at P10, P21, and P40, primarily around the neuronal cell bodies and proximal dendritic processes. (b) In rat neocortex, immunofluorescence double labeling with NeuN (red) and SV2A (green) demonstrated a similar perisomatic and dendritic neuronal expression that increased progressively from P10 to P40. (c) Western blot quantification of SV2A levels in the postnatal rat hippocampus showed a significant age-dependent increase $(P<0.001$; one-way ANOVA). SV2A was already expressed at P5, P10, and P14 but at significantly lower levels than at P21 and P40 $(P<0.05)$. (d) Western blot analysis of SV2A in the rat neocortex at the same postnatal ages demonstrated a similar significant increase from P5 to P40 ( $P<0.05$; one-way ANOVA). Bars in $\mathbf{a}$ and $\mathbf{b}$ represent $10 \mu \mathrm{m} .{ }^{*} P<$ 0.05 . $P$, postnatal day; SV2A, synaptic vesicle protein $2 A$.

In relation to the protection against increased seizure susceptibility to the "second-hit" KA, we next examined whether LEV treatment also protected against KA-induced neuronal injury. In rats receiving KA at P40, we counted the total number of Fluoro-Jade B-positive cells in hippocampal CA3 subfield on sections from stereotactically identical regions (24). We found a significant reduction in the number of FluoroJade B-positive neurons in the CA3 region in LEV-treated HS rats, as compared with vehicle-treated HS animals subsequently exposed to KA $(1.55 \pm 0.83, n=5$, vs. $9.75 \pm 2.91, n=$ $6, P=0.035$; Figure 1f,g).

\section{Developmental Expression of SV2A Protein in Rat Hippocampus and Neocortex}

To determine the presence of the putative LEV target in the neonatal brain, we performed immunofluorescent double labeling with NeuN and SV2A at P10, P21, and P40. We found that in both the hippocampus and neocortex, SV2A was present and localized to neuronal cells, primarily in dendritic processes (Figure 2a,b). Western blot analysis of hippocampal and neocortical homogenates demonstrated that SV2A was expressed
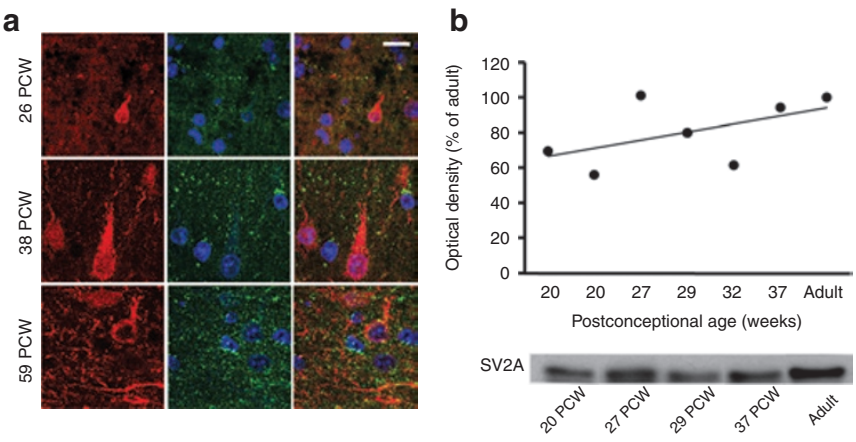

Figure 3. Age-specific differences in SV2A expression in the human perinatal neocortex. (a) Human neocortical sections, double-labeled with MAP2 (red) and SV2A (green), and nuclear staining with 4',6diamidino-2-phenylindole (blue), demonstrated a small number of SV2Aimmunolabeled puncta among the scanty-branched neurons at 26 postconceptional wk (PCW), but more immunolabeled puncta in the neuropil and close to the neuronal perikarya at 38 and 59 PCW. (b) Western blot analysis of human neocortex specimens aged 20-37 PCW showed lower levels of SV2A at midgestation (20 PCW) and slightly higher expression in preterm (27-32 PCW) and near-term (37 PCW) newborns $\left(r^{2}=0.2838\right)$. Bar in a represents $10 \mu \mathrm{m}$. SV2A, synaptic vesicle protein $2 \mathrm{~A}$.

at all ages examined (Figure 2c,d), consistent with the immunohistochemistry results. SV2A levels were lower at P5, P10, and P14 as compared with older ages in both the hippocampus $(P<0.001)$ and neocortex $(P<0.05)$. At $\mathrm{P} 10$, SV2A expression reached approximately $43 \%$ of adult (P40) levels in the hippocampus (Figure 2c) and $46 \%$ of adult levels in the neocortex (Figure 2d).

\section{Expression of SV2A in the Developing Human Neocortex}

To validate the expression of SV2A target protein in humans, we performed immunofluorescence double labeling of postmortem human neocortex. Similar to rats, human neocortex demonstrated that SV2A was already present in the neuropil at 26 postconceptional wk (PCW) (Figure 3a). The density of SV2A-positive puncta, located mostly in the close vicinity of MAP-2-positive neuronal cell bodies and dendrites, increased substantially at 38 and 59 PCW. Western blot analysis of human neocortex specimens aged 20-37 PCW (Figure 3b) showed the same developmental regulation. SV2A was lowest at midgestation ( $62 \%$ of adult values at $20 \mathrm{PCW})$, increasing through the preterm period (81\% of adult between 27 and 32 PCW), and reaching almost adult values near term birth (94\% of adult at $37 \mathrm{PCW}$ ).

\section{DISCUSSION}

These experiments provide first evidence for both prophylactic anticonvulsant and disease-modifying efficacy of LEV in a clinically relevant model of neonatal seizures. In addition, these results validate the neuronal expression of the putative target of LEV, the SV2A in immature rat (6) and human brain.

Our model of acute hypoxia-induced seizures at $\mathrm{P} 10$ recapitulates epileptogenic and clinical phenomena of neonatal epilepsy exhibiting not only early refractory seizures $(23,27)$ but also long-term effects including increased seizure susceptibility and hippocampal hyperexcitability $(21,22,28,29)$, enhanced 
seizure-induced neuronal injury $(21,22)$, and development of spontaneous seizures (24), cognitive deficits $(25,30)$, and autistic-like behavior (31). A brief episode of moderate global hypoxia at P10-12 induces spontaneous tonic-clonic seizures in rat pups. After a latent period of $1-2 \mathrm{wk}$, rats develop spontaneous recurrent seizures, which progress over time such that in adulthood, epilepsy is present in approximately $95 \%$ of animals (24). Of note, HSs do not induce neuronal death but result in hippocampal CA3 mossy fiber sprouting $(22,24)$ and in long-term functional changes in hippocampal CA1 neurons, including a-amino-3-hydroxy-5-methyl-4-isoxazolepropionic acid (AMPA)-type glutamate receptor potentiation $(29,31,32)$, downregulation of GABA-ergic inhibition (33), and impaired synaptic plasticity (32), which may provide a basis for the longterm neurobehavioral deficits.

Hypoxia-induced seizure activity in this model critically depends on the activation of the AMPA receptors, because it is selectively blocked by AMPA receptor antagonists but not by $N$-methyl-D-aspartate-type glutamate receptor blockers or AEDs that increase inhibitory GABA receptor function $(21,26,27)$. Of note, AMPA receptor antagonists are able to prevent the enhanced seizure susceptibility and seizure-induced hippocampal injury $(21,22,26,29)$, to attenuate the increases in AMPA receptor function (29) and to preserve synaptic plasticity (32), even when administered after HSs.

Here, we show that LEV effectively suppressed acute behavioral and EEG seizures induced by perinatal hypoxia in a dose-dependent fashion, with both the 25- and 50-mg/kg doses affording similar superior protection as compared with the lower $10-\mathrm{mg} / \mathrm{kg}$ dose. These doses have been shown to be within a range similar to that used in humans (34) and that yield serum levels of $20-50 \mu \mathrm{g} / \mathrm{ml}$ (35). Furthermore, the acute LEV protection in this model was comparable with that previously reported for AMPA receptor antagonists (about 75-87\% acute seizure suppression for topiramate and talampanel $(21,26)$ vs. $63.6 \%$ for LEV in this study).

LEV treatment at P10 prevented the enhanced susceptibility to "second-hit" KA-induced seizures and hippocampal neuronal injury at P40. These results are the first to suggest that in addition to acute seizure suppression, LEV may have longer-lasting protective effects in later life following neonatal seizures (15-18). LEV treatment resulted in more than $80 \%$ reduction in KA-induced neuronal death, which is comparable with the effects of AMPA receptor antagonists ( $60 \%$ reduction in both pre- and posttreatment paradigms) $(21,22,26)$. Given that LEV was administered 60 min before seizure induction, this initial study does not fully elucidate whether the long-term protection is due mainly to acute seizure suppression. However, the present treatment paradigm is likely to be acting following seizure induction. LEV functions differently from neurotransmitter receptor antagonists, which have instant effects upon crossing the blood-brain barrier. We chose the 60-min pretreatment paradigm on the basis of previous in vitro electrophysiology data documenting a significant delay (at least $30 \mathrm{~min}$ ) before LEV develops its full inhibitory effect on synaptic transmission (7). Because SV2A protein is localized within the intracellular compartment, LEV must enter the neuron in order to influence synaptic transmission. Indeed, as compared with LEV, the AMPA receptor antagonist 6-cyano-7-nitroquinoxaline-2,3-dione (CNQX), which acts on surface receptors, exerts a more rapid effect than LEV (7). Existing data from adult animal epilepsy models demonstrate a potent antiepileptogenic action for LEV, in addition to its acute antiepileptic effects (10-13). Further investigation of LEV as a postseizure treatment paradigm is needed to definitively determine whether LEV can have protective efficacy independent of an effect on the acute seizures.

Previous animal studies by others $(19,20)$ demonstrated that LEV is devoid of a proapoptotic action in the infant brain even at significantly higher doses $(1,500 \mathrm{mg} / \mathrm{kg})$ than that used in the current study, whereas older AEDs such as phenytoin, valproate, and phenobarbital induce substantial region-specific apoptotic neuronal death (36). It is speculated that early childhood seizures may have deleterious long-term effects by preventing normal brain development, but the fact that several AEDs may induce excessive neuronal cell death raises concern about whether the AED therapy may also be detrimental. Our results suggest that doses of LEV that are effectively anticonvulsant in a neonatal seizure model are in a range that has been shown to be safe and devoid of any apoptotic effects in the immature rodent brain (19). It will be important to evaluate the long-term consequences of LEV therapy in infancy through adulthood by examining behavioral outcomes and seizure susceptibility in epileptic neonates exposed to LEV. Clinical retrospective studies suggest that LEV monotherapy is safe and effective in a wide range of epilepsies in neonates, infants, and children younger than $4 \mathrm{y}(15,37)$.

Here, we also demonstrate that the putative target SV2A protein is expressed in the hippocampus and neocortex of immature rats and localized to neurons, primarily on dendritic processes. Western blot analysis of rat hippocampus and neocortex confirmed the expression of SV2A at all ages and demonstrated a gradual increase with development. Previous studies have shown that SV2A mRNA is already highly expressed at embryonic day 17 , well before neurons make their first synaptic contacts (6). However, the developmental expression of SV2A protein has not been systematically evaluated, which is more clinically relevant, given that this is the putative target of LEV. Our developmental time course data, in agreement with our in vivo effects and previous studies showing that LEV is effective in suppressing recurrent ictal-like activity in in vitro preparations from neonatal rats (38), supports SV2A involvement in mechanisms resulting in susceptibility to epileptogenesis during neonatal life.

Furthermore, we show that SV2A protein is expressed in the neurons in the neonatal human brain. Similar to the earlier ages in the rodent, there was lower expression of SV2A at midgestation, which increased through the preterm period to reach near-adult levels in the term infant, a profile consistent with the developmental expression of other synaptic proteins, such as synaptophysin and postsynaptic density-95 (39). Although SV2A immunoreactivity has been reported in patients with epilepsy (40), ours is the first study to document its expression in term normal human brain. The expression of SV2A in specimens of 
focal cortical dysplasia and tuberous sclerosis complex cortical tubers has been recently described in patients aged 5-40 y (40). Similar to our results, in control specimens, SV2A immunoreactivity was seen throughout all the cortical layers, with punctate labeling around the soma and dendrites of neurons. In specimens of focal cortical dysplasia and tuberous sclerosis complex cortical tubers, there was reduced neuropil expression and altered cellular and subcellular distribution, perhaps indicating a possible contribution of SV2A to the epileptogenicity of these malformations. Taken together with the preclinical anticonvulsant and antiepileptogenic efficacy and lack of neurotoxicity at supratherapeutic doses, these data support continued interest in a clinical trial of parenterally administered LEV for neonatal seizures.

\section{METHODS}

\section{Animals}

Male Long-Evans rats (Charles River Laboratories, Wilmington, MA) were housed in a temperature-controlled animal facility with a 12-h light/dark cycle. All rodent experiments were approved by the Animal Care and Use Committee at the Children's Hospital (Boston, MA) and were in accordance with the guidelines of the Committee on Care and Use of Laboratory Animals, Institute of Laboratory Animals Resources.

\section{Rat Model of Hypoxia-Induced Seizures}

At P10, rat pups were exposed to global hypoxia for $15 \mathrm{~min}$ in an airtight chamber into which $\mathrm{N}_{2}$ was rapidly infused, as described previously $(23,24)$. The oxygen concentration was maintained at $7 \%$ for $8 \mathrm{~min}, 5 \%$ for $6 \mathrm{~min}$, and $4 \%$ for $1 \mathrm{~min}$ before termination of hypoxia. Normoxic littermate controls were left at room air separated from the dam. The body temperature of all pups was maintained at $32-34{ }^{\circ} \mathrm{C}$ on a warming blanket. All rats were returned to their dams within $30 \mathrm{~min}$ after the experiment.

\section{LEV Treatments}

Intraperitoneal injections of either LEV $(10,25$, or $50 \mathrm{mg} / \mathrm{kg})$ or vehicle (phosphate-buffered saline) were administered $60 \mathrm{~min}$ before hypoxia. LEV was synthesized in the chemical laboratory of UCB Pharma, Braine-l'Alleud, Belgium.

\section{Video-EEG Recordings With Implanted Subcutaneous Electrodes} During hypoxia, rat behavior was recorded by video monitoring by an investigator blinded to treatment group. EEG activity was simultaneously recorded from subcutaneous electrodes (Ives EEG Solutions, Manotick, Canada) that were placed in the dorsal scalp: one wire at midline over the olfactory bulb and two wires over the left and right parietal regions. Electrocardiograms were obtained from an electrode placed subcutaneously on the dorsal trunk. Video-EEGs were recorded using the Stellate Harmonie version 6.0 (Stellate, Montreal, Canada) (24).

\section{HSs Monitoring and Analysis}

Behavior was scored by video review by a blinded investigator, and total tonic-clonic seizure duration during hypoxia was compared between treatment groups. EEG and electrocardiogram activities were recorded for $150 \mathrm{~min}$ (baseline for $15 \mathrm{~min}$, between drug injection and induced hypoxia for $60 \mathrm{~min}$, during hypoxia for $15 \mathrm{~min}$, and after hypoxia for $60 \mathrm{~min}$ ). Cumulative duration of ictal activity during the 15-min hypoxia was analyzed by a researcher blinded to the treatment paradigm and compared between groups. An EEG seizure was defined by paroxysmal, rhythmic discharges displaying high-amplitude diffuse fast activity lasting more than $3 \mathrm{~s}$ in duration. These events were accompanied by tonic-clonic seizures, automatisms followed by head and limb movements, and myoclonic jerks (24). Data for the LEV-treated groups were expressed as a percentage of the mean total duration of seizures for the paired vehicle-treated group (100\%). Differences in seizure duration among groups were analyzed by one-way ANOVA followed by multiple comparison procedures (Bonferroni $t$-tests). Statistical significance was established at $P<0.05$.

\section{KA-Induced Seizures}

A subsequent "second-hit" experiment was performed to assess the effects of LEV on the time course of delayed epileptogenesis following neonatal hypoxia. Susceptibility of rats to KA-induced seizures was assessed $30 \mathrm{~d}$ (P40) after they were exposed to hypoxia at P10. KA (10 mg/kg; Cayman Chemical, Ann Arbor, MI) was injected intraperitoneally, and seizure activity was video recorded over $3 \mathrm{~h}$. The latency to onset of first behavioral seizure (repetitive pawing and wet-dog shake) and seizure severity (maximal seizure stage reached graded from 0 to 5) were evaluated in a blinded manner, as described previously (22). For statistical analysis, Kruskal-Wallis and Mann-Whitney $U$ tests were used, and significance was assumed for $P<0.05$.

\section{Fluoro-Jade B Procedures}

Rats were killed $72 \mathrm{~h}$ after KA injections by transcardiac perfusion with phosphate-buffered saline followed by $4 \%$ paraformaldehyde. Brains were postfixed in the same fixative overnight and then cryoprotected in $30 \%$ sucrose. Serial coronal sections were cut at $16 \mu \mathrm{m}$ on a Leica Cryostat CM 3050S (Leica Microsystems, Nussloch, Germany). Staining for Fluoro-Jade B/AG310 (Millipore, Temecula, CA) was performed as previously described (24). Counts of positively stained cells in the hippocampal subfield CA3 were used to quantify neuronal death $(22,24,26)$. Statistical significance between vehicletreated and LEV-treated groups was established at $P<0.05$, using the unpaired $t$-test.

\section{Human Subjects}

Autopsy parietal-occipital cortex specimens ( $n=15 ; 8$ males/ 7 females) were obtained from the Children's Hospital and Brigham and Women's Hospital, Boston. The study has been approved by the hospitals' institutional review boards (Children's Hospital and Partners Healthcare System, Boston, MA), and informed consent has been obtained in all cases. The age of the subjects ranged from midgestation through to adulthood. All ages were expressed as PCW, calculated as gestational age plus postnatal age, with the exception of the adult cases, for which age was expressed in years. All patients died from nonneurological causes. In infants, the cause of death included stillbirth $(n=1)$, prematurity $(n=4)$, congenital cardiovascular defects $(n=6)$, and necrotizing enterocolitis $(n=2)$. In adulthood, death was due to hematologic malignancies $(n=2)$. The postmortem interval was less than $24 \mathrm{~h}$ in all cases (mean $=18.7 \mathrm{~h}$ ), and only well-preserved tissue was included in the study. No brain abnormalities or lesions were observed by macroscopic neuropathologic diagnostic examination, and except for two patients presenting mild diffuse white matter gliosis, all cases were diagnosed as histologically normal (by R.D.F.).The relevant clinical and neuropathological data of human brain specimens used in this study are summarized in Supplementary Table S1 online.

\section{Immunohistochemistry}

Rat brains were perfused, fixed, and cryoprotected as described above, and $16-\mu \mathrm{m}$ coronal sections were cut at the anterior, middle, and posterior hippocampus levels. Sections were blocked and incubated overnight at $4^{\circ} \mathrm{C}$ with a mouse monoclonal antibody to Neuronal Nuclei NeuN/MAB377 (1:100; Millipore), followed by fluorescent goat Alexa Fluor 568 antimouse immunoglobulin G (1:1,000; Molecular Probes, Eugene, OR) secondary antibody incubation. Double labeling was performed sequentially with a rabbit polyclonal antibody to SV2A/ ab32942 (1:100; Abcam, Cambridge, MA) and Oregon Green 488 antirabbit immunoglobulin G (1:1,000; Molecular Probes). Slides were coverslipped with an antifade medium (Fluoromount-G, Southern Biotechnology, Birmingham, AL) (31). Confocal fluorescence images were taken on a scanning laser microscope (LSM 510 META NLO, Carl Zeiss MicroImaging, Thornwood, NY) with a $63 \times$ objective (scan zoom 2.0). Human tissue immunohistochemistry was performed on paraffin-embedded specimens (age: $26-137 \mathrm{PCW}, n=7$ ). Tissue was cut at $5 \mu \mathrm{m}$ and preserved at room temperature until use. Deparaffination of sections was performed through double 10-min xylene immersion and progressive rehydration in a series of ethanol solutions. Sections were immersed in an antigen retrieval solution $(0.1 \mathrm{~mol} / \mathrm{l}$ citrate buffer, $\mathrm{pH}=6.0$ ) in a microwave at $95^{\circ} \mathrm{C}$ for $10 \mathrm{~min}$. After cooling, sections were blocked and incubated overnight at $4^{\circ} \mathrm{C}$ in primary antibodies 
(mouse monoclonal antibody to SV2A/ab49572 (1:1,000, Abcam) and rabbit polyclonal antibody to microtubule-associated protein 2MAP2/ AB5622 (1:750, Millipore)) and then incubated with the same secondary antibodies used in the rat. Slides were coverslipped with Vectashield containing the nucleic acid stain 4',6-diamidino-2-phenylindole (Vector Laboratories, Burlingame, CA) and examined using a spinning disc fluorescence microscope (Eclipse Ti, Nikon Instruments, Melville, NY). Multiple plane images, collected at $0.5 \mu \mathrm{m}$ intervals, were captured to acquire z-stacks. Control sections were incubated with the omission of one or both primary antibodies. Qualitative analysis was performed on two to three sections per brain.

\section{Western Blot Analysis}

Rat brains were quickly removed, and either cortex or hippocampus samples were separated and rapidly frozen in cold ethanol and stored at $-80^{\circ} \mathrm{C}$ until used for whole cell preparation ( $n=3-5$ samples per age group). Tissue was homogenized on ice in lysis buffer containing one Complete Mini Protease Inhibitor Cocktail Tablet per $10 \mathrm{ml}$ of buffer and the phosphatase inhibitors phenylmethanesulfonyl fluoride ( $1 \mathrm{mM})$, sodium orthovanadate $(1 \mathrm{mmol} / \mathrm{l})$, and okadaic acid $(0.1 \mathrm{mM})$ (Roche, Indianapolis, IN). Total protein amounts were measured using the Bradford protein assay (Bio-Rad, Hercules, CA), and samples were diluted for equal amounts of protein in each sample. Samples were run on $4-20 \%$ gradient Tris-HCl gel (Bio-Rad) and transferred onto polyvinylidene difluoride membranes, blocked in 5\% nonfat dry milk and incubated overnight at $4^{\circ} \mathrm{C}$ in rabbit polyclonal antibody to SV2A/ab32942 (1:500; Abcam). Horseradish peroxidase-conjugated antirabbit immunoglobulin G/no. 1858415 (1:10,000; Pierce Biotechnology, Rockford, IL) were used as secondary antibodies. Protein bands were visualized by chemiluminescence using the Image Reader LAS 3000 digital system and Image Gauge 4.0 software (Fuji Film Life Sciences, Stamford, CT). Blots were stripped and reprobed with a mouse monoclonal antibody to actin $(0.1 \mathrm{mg} / \mathrm{ml}$ SigmaAldrich, St Louis, MO). The optical density of each band was normalized to the corresponding actin band, and group means for each age were calculated for statistical analysis. Differences in mean protein levels were analyzed by one-way ANOVA flowed by multiple comparison procedures (Bonferroni $t$-tests). Statistical significance was established at $P<0.05$ (31). Fresh-frozen autopsy human brain tissue samples (age: 20 PCW to $43 \mathrm{y}, n$ $=8$ ) were processed in a similar fashion. Equal protein load was estimated using staining with Ponceau S/P7170 solution according to the manufacturer's instructions (Sigma Aldrich). Data for each age were expressed as percentage of adult mean (100\%). Regression analysis of postnatal age on relative SV2A values was performed, and a linear regression curve was fitted to the individual SV2A levels.

\section{SUPPLEMENTARY MATERIAL}

Supplementary material is linked to the online version of the paper at http:// www.nature.com/pr

\section{ACKNOWLEDGMENTS}

The authors thank Dr Rakhade for his advice regarding quantitative electroencephalography analysis.

\section{STATEMENT OF FINANCIAL SUPPORT}

Partial funding for this research was obtained as an unrestricted, researcherinitiated grant from UCB Pharma, Braine-l'Alleud, Belgium. This work was also supported by National Institutes of Health grant nos. NS 31718 (from the National Institute of Neurological Disorders and Stroke, to F.E.J.), DP1 OD003347 (from the Office of Director, to F.E.J.), and P30 HD 18655 (from the Intellectual Developmental Disabilities Center, National Institute of Child Health and Human Development).

Disclosure: The authors declared no conflict of interest.

\section{REFERENCES}

1. Silverstein FS, Jensen FE. Neonatal seizures. Ann Neurol 2007;62:112-20.

2. Sankar R, Painter MJ. Neonatal seizures: after all these years we still love what doesn't work. Neurology 2005;64:776-7.

3. Glauser TA, Ayala R, Elterman RD, et al. Double-blind placebo-controlled trial of adjunctive levetiracetam in pediatric partial seizures. Neurology 2006;66:1654-60.
4. Khurana DS, Kothare SV, Valencia I, Melvin JJ, Legido A. Levetiracetam monotherapy in children with epilepsy. Pediatr Neurol 2007;36:227-30.

5. Kaminski RM, Matagne A, Leclercq K, et al. SV2A protein is a broadspectrum anticonvulsant target: functional correlation between protein binding and seizure protection in models of both partial and generalized epilepsy. Neuropharmacology 2008;54:715-20.

6. Bajjalieh SM, Frantz GD, Weimann JM, McConnell SK, Scheller RH. Differential expression of synaptic vesicle protein 2 (SV2) isoforms. J Neurosci 1994;14:5223-35.

7. Yang XF, Rothman SM. Levetiracetam has a time- and stimulationdependent effect on synaptic transmission. Seizure 2009;18:615-9.

8. Madeja M, Margineanu DG, Gorji A, et al. Reduction of voltage-operated potassium currents by levetiracetam: a novel antiepileptic mechanism of action? Neuropharmacology 2003;45:661-71.

9. Lukyanetz EA, Shkryl VM, Kostyuk PG. Selective blockade of N-type calcium channels by levetiracetam. Epilepsia 2002;43:9-18.

10. Löscher W, Hönack D, Rundfeldt C. Antiepileptogenic effects of the novel anticonvulsant levetiracetam (ucb L059) in the kindling model of temporal lobe epilepsy. J Pharmacol Exp Ther 1998;284:474-9.

11. Stratton SC, Large CH, Cox B, Davies G, Hagan RM. Effects of lamotrigine and levetiracetam on seizure development in a rat amygdala kindling model. Epilepsy Res 2003;53:95-106.

12. Yan HD, Ji-qun C, Ishihara K, Nagayama T, Serikawa T, Sasa M. Separation of antiepileptogenic and antiseizure effects of levetiracetam in the spontaneously epileptic rat (SER). Epilepsia 2005;46:1170-7.

13. Vinogradova LV, van Rijn CM. Anticonvulsive and antiepileptogenic effects of levetiracetam in the audiogenic kindling model. Epilepsia 2008;49:1160-8.

14. Lamberty Y, Margineanu DG, Klitgaard H. Absence of negative impact of levetiracetam on cognitive function and memory in normal and amygdalakindled rats. Epilepsy Behav 2000;1:333-42.

15. Khan O, Chang E, Cipriani C, Wright C, Crisp E, Kirmani B. Use of intravenous levetiracetam for management of acute seizures in neonates. Pediatr Neurol 2011;44:265-9.

16. Abend NS, Gutierrez-Colina AM, Monk HM, Dlugos DJ, Clancy RR. Levetiracetam for treatment of neonatal seizures. J Child Neurol 2011;26:465-70.

17. Ramantani G, Ikonomidou C, Walter B, Rating D, Dinger J. Levetiracetam: safety and efficacy in neonatal seizures. Eur J Paediatr Neurol 2011;15:1-7.

18. Fürwentsches A, Bussmann C, Ramantani G, et al. Levetiracetam in the treatment of neonatal seizures: a pilot study. Seizure 2010;19:185-9.

19. Kim JS, Kondratyev A, Tomita Y, Gale K. Neurodevelopmental impact of antiepileptic drugs and seizures in the immature brain. Epilepsia 2007;48:Suppl 5:19-26.

20. Manthey D, Asimiadou S, Stefovska V, et al. Sulthiame but not levetiracetam exerts neurotoxic effect in the developing rat brain. Exp Neurol 2005;193:497-503.

21. Koh S, Jensen FE. Topiramate blocks perinatal hypoxia-induced seizures in rat pups. Ann Neurol 2001;50:366-72.

22. Koh S, Tibayan FD, Simpson JN, Jensen FE. NBQX or topiramate treatment after perinatal hypoxia-induced seizures prevents later increases in seizure-induced neuronal injury. Epilepsia 2004;45:569-75.

23. Jensen FE, Applegate CD, Holtzman D, Belin TR, Burchfiel JL. Epileptogenic effect of hypoxia in the immature rodent brain. Ann Neurol 1991;29:629-37.

24. Rakhade SN, Klein PM, Huynh T, et al. Development of later life spontaneous seizures in a rodent model of hypoxia-induced neonatal seizures. Epilepsia 2011;52:753-65.

25. Jensen FE, Holmes GL, Lombroso CT, Blume HK, Firkusny IR. Age-dependent changes in long-term seizure susceptibility and behavior after hypoxia in rats. Epilepsia 1992;33:971-80.

26. Aujla PK, Fetell MR, Jensen FE. Talampanel suppresses the acute and chronic effects of seizures in a rodent neonatal seizure model. Epilepsia 2009;50:694-701.

27. Jensen FE, Blume H, Alvarado S, Firkusny I, Geary C. NBQX blocks acute and late epileptogenic effects of perinatal hypoxia. Epilepsia 1995;36: 966-72. 
28. Jensen FE, Wang C. Hypoxia-induced hyperexcitability in vivo and in vitro in the immature hippocampus. Epilepsy Res 1996;26:131-40.

29. Rakhade SN, Zhou C, Aujla PK, Fishman R, Sucher NJ, Jensen FE. Early alterations of AMPA receptors mediate synaptic potentiation induced by neonatal seizures. J Neurosci 2008;28:7979-90.

30. Mikati MA, Zeinieh MP, Kurdi RM, et al. Long-term effects of acute and of chronic hypoxia on behavior and on hippocampal histology in the developing brain. Brain Res Dev Brain Res 2005;157:98-102.

31. Talos DM, Sun H, Zhou X, et al. The interaction between early life epilepsy and autistic-like behavioral consequences: a role for the mammalian target of rapamycin (mTOR) pathway. PLoS ONE 2012;7:e35885.

32. Zhou C, Lippman JJ, Sun H, Jensen FE. Hypoxia-induced neonatal seizures diminish silent synapses and long-term potentiation in hippocampal CA1 neurons. J Neurosci 2011;31:18211-22.

33. Sanchez RM, Dai W, Levada RE, Lippman JJ, Jensen FE. AMPA/kainate receptor-mediated downregulation of GABAergic synaptic transmission by calcineurin after seizures in the developing rat brain. J Neurosci 2005;25:3442-51.

34. Merhar SL, Schibler KR, Sherwin CM, et al. Pharmacokinetics of levetiracetam in neonates with seizures. J Pediatr 2011;159:152-154.e3.
35. van Vliet EA, Aronica E, Redeker S, Boer K, Gorter JA. Decreased expression of synaptic vesicle protein $2 \mathrm{~A}$, the binding site for levetiracetam, during epileptogenesis and chronic epilepsy. Epilepsia 2009;50: 422-33.

36. Bittigau P, Sifringer M, Ikonomidou C. Antiepileptic drugs and apoptosis in the developing brain. Ann N Y Acad Sci 2003;993:103-14; discussion $123-4$.

37. Grosso S, Cordelli DM, Franzoni E, et al. Efficacy and safety of levetiracetam in infants and young children with refractory epilepsy. Seizure 2007;16:345-50.

38. Quilichini PP, Diabira D, Chiron C, Milh M, Ben-Ari Y, Gozlan H. Effects of antiepileptic drugs on refractory seizures in the intact immature corticohippocampal formation in vitro. Epilepsia 2003;44:1365-74.

39. Glantz LA, Gilmore JH, Hamer RM, Lieberman JA, Jarskog LF. Synaptophysin and postsynaptic density protein 95 in the human prefrontal cortex from mid-gestation into early adulthood. Neuroscience 2007;149:582-91.

40. Toering ST, Boer K, de Groot M, et al. Expression patterns of synaptic vesicle protein $2 \mathrm{~A}$ in focal cortical dysplasia and TSC-cortical tubers. Epilepsia 2009;50:1409-18. 